\title{
CUMULATIVE AUTHOR INDEX
}

\section{(Volume 9)}

Adamchuk, V. K. 1375

Aebi, P. 235

Afanas'ev, I. M. 491

Agui, A. 843, 849, 1327

Aguilar-Frutis, M. 1637

Aita, O. 871, 1035

Aiura, Y. 1085

Akbar, A. A. 1459

Akimitsu, J. 1097

Akinaga, H. 331

Aksela, H. 137

Aldao, C. M. 1803

Alivisatos, A. P. 437

Allen, J. W. 1251, 1309

Alonso, C. F. 1709

Alonso, J. A. 1121

Altarellli, M. 1167

Alvarado-Leyva, P. G. 1747

Álvarez, J. 117, 147

Alvarez-Fregoso, O. 1637

Amarantov, S. V. 1339

Andersen, T. H. 723

Anderson, E. 177

Ando, M. 631

Andreev, E. P. 491

Andreić, Ž. 627

Arai, A. 663

Araki, T. 441

Arbey-Rodriguez, J. 1815

Arenholz, E. 549

Arias, D. 1611

Ariosa, D. 1109

Arita, M. 529, 535, 1079

Arranz, A. 729

Aryasetiawan, F. 1281

Asensio, M. C. 729

Asokan, K. 1053

Attili, A. 541

Augustsson, A. 1369

Auwärter, W. 1243

Avakyan, S. V. 491

Avendaño, C. 1827

Avila, J. 729

Azofeifa, D. E. 1721

Baba, Y. 77

Baca, E. 1611, 1729

Bader, S. D. 1611

Balasubramanian, T. 687, 1493

Baldwin, K. G. H. 31

Baltenkov, A. S. 1143

Banfi, G. 541
Bansmann, J. 913

Baraldi, A. 741

Barbo, F. 249

Barinov, A. 413, 705, 751

Bartolo-Pérez, P. 1709, 1779

Bauer, E. 171, 223, 1485

Baumvol, I. J. R. 393

Beamson, G. 1661

Bech, L. 723

Bechstedt, F. 1497

Beetz, T. 185

Berger, A. 1611

Berger, H. 1109, 1117

Bernardinello, A. 347

Bernstorff, S. 455

Bertolo, M. 249

Bezmelnitsyn, V. N. 1339

Bianchi, A. D. 1309

Bianco, A. 249

Biasiol, G. 413

Björneholm, O. 3

Blaha, P. $1029,1243,1275,1281$

Blasco, J. 821, 1071

Boltalina, O. V. 1339

Bonanni, B. 413

Bondino, F. 741

Bondybey, V. E. 353

Boody, F. P. 347

Bostedt, C. 387

Bourgeois, S. 949

Bovet, M. 235

Bower, C. 431

Bowler, M. 577

Bradshaw, A. M. 223

Brandão, R. 393

Braun, J. 883

Bressler, P. 359

Bruno, F. 937

Bureev, O. A. 1345

Burillo, G. 1637

Butorin, S. M. 989

Cai, Y. Q. 907

Caicedo, L. M. 1693

Calderón, C. 1617

Caldwell, C. D. 1229

Callcott, T. A. 1345

Camacho B., A. S. 1623

Campillo, G. 1611

Campos, A. M. 1631

Campuzano, J. C. 1127

Casais, M. T. 1121
Castaño, V. M. 1795, 1799

Castro, L. F. 1611, 1729

Cattani, M. 1409

Cautero, G. 249, 541

Cejnarová, A. 347

Cepek, C. 775

Chandesris, D. 949

Chang, C. F. 1007

Chang, C. H. 497

Chang, H. W. 1109

Chang, Y. K. 293

Chassé, A. 901, 1173

Chassé, T. 359, 381

Chavez-Ramirez, J. 1637

Chen, C. T. 1007

Chen, J. 1007

Chen, Y. Y. 293

Cherifi, S. 171

Chernenko, A. S. 347

Chernysheva, L. V. 1155, 1209

Chiaradia, P. 1497

Chiba, H. 93

Chim, R. Y. L. 129, 153

Chiou, J. W. 293, 1053

Cho, B. 285

Cho, T. 555

Choi, K. 255

Chuang, T. J. 213

Chuang, T. M. 1109

Chung, S. C. 1007

Chung, S. 285

Chvostová, D. 347, 473

Cimino, R. 249

Claessen, R. 1029, 1275, 1281

Clark, N. 1721

Cocoletzi, G. H. 1641, 1651

Collins, D. J. 129

Comelli, G. 541, 741

Connerade, J. P. 39

Coreno, M. 159

Cornelius, S. 693

Corona-Organiche, E. 1645

Cota-Araiza, L. 1697

Cotzomi-Paleta, J. 1641

Couprie, M. E. 599

Coyotécatl, H. A. 1651

Cuamatzi, M. 1715

Cvetko, D. 709

Czasch, A. 93

D'Addato, S. 709

Daliang, S. 1395 
Danailov, M. B. 1339

Danger, J. 949

da Rosa, E. B. O. 393

de Abajo, F. J. G. 1213

De Anda, F. 1741

de Fanis, A. 51

de Melo, O. 1725

de Oliveira, N. 655

de Simone, M. 159

del Castillo-Mussot, M. 1651, 1827

Delaunay, R. 921, 977

Demeter, M. 1097

Denecke, R. 789, 797

Denlinger, J. D. 1251, 1309

Desnica, U. V. 455

Desnica-Frankovic, I. D. 455

Dhalenne, G. 1103, 1369

di Fabrizio, E. 243

di Fonzo, S. 925

Díaz, G. 1779

Díaz, T. 1715, 1741

Díaz-Reyes, J. 1645

Ding, H. 1127

Doi, Y.-I. 319

Dolmatov, V. K. 39, 1143

Domashevskaya, E. P. 1047

Dooley, P. M. 31

Downes, J. E. 1369

Dreiner, S. 735

Drube, W. 949

Dubcek, P. 455

Duda, L.-C. 1103, 1369

Dudin, P. V. 1339

Dunwoody, P. M. 277

Duque, C. A. 1753

Eberhardt, W. 461

Ebert, H. 1135, 1179

Ecker, M. 759

Edamoto, K. 717

Ederer, D. L. 1345

Eguchi, R. 983

Ehresmann, A. 57

Eickhoff, T. 949

Eisebitt, S. 461

Ejima, T. 521, 587, 943

Ejiri, A. 447

el Mellouhi, F. 775

El-Hassan, S. A. 1379

Elizondo-Villarreal, N. 1661

Ellwi, S. S. 627

Engel, W. 223

Erkoç, Ş. 1401

Erman, P. 117

Esch, F. 741

Escobosa, A. 1741

Fadley, C. S. 1213
Falcony, C. 1637

Falkenberg, G. 1809

Farias, M. H. 1661, 1697, 1709

Fayard, B. 203

Fecher, G. H. $\quad$ 509, 883, 889, 895

Fedorov, A. V. 1091

Fedorova, T. A. 1185

Felfli, Z. 1155

Fell, B. 577

Feng, J. 549

Ferrini, G. 541

Feser, M. 185

Feulner, P. 759

Fink, R. 223

Fisk, Z. 1309

Flavell, W. R. 277, 577

Flesch, R. 99, 105, 141

Floreano, L. 709, 937

Föhlisch, A. 759

Follath, R. 165

Fondacaro, A. 541

Fonseca, K. 1785

Fonseca, L. F. 1655

Fontana, S. 249

Franciosi, A. 249

Frankowski, M. 353

Freund, H.-J. 223

Frost, F. 381

Fu, Z. X. 699

Fujikawa, C. 635

Fujikawa, T. 901

Fujiki, T. 313

Fujimori, A. 1065

Fujimori, S.-I. 1065

Fujimoto, H. 441

Fujino, H. 529, 1079

Fujisawa, H. 1041

Fukui, K. 319

Furudate, M. 571

Furukawa, T. 555

Gáal, R. 1109

Galván, D. H. 1779

García-Rocha, M. 1667

García, J. 821, 1071

Garcia, M. 1637

Garcia-Mendez, M. 1661

Garreau, Y. 1497

Garzella, D. 599

Gasparov, L. V. 1109

Gatto, A. 599

Gauthier, Y. 1431

Gavioli, L. 1117

Gesari, S. B. 1437

Gesland, J. Y. 621

Gewinner, G. 1431

Giannessi, L. 599

Gibson, S. T. 31
Giovanardi, C. 937

Giraldo, J. C. 1765

Giraldo, J. J. 1631, 1703

Giratá, D. 1729

Gminder, E. 45

Gnatchenko, E. V. 651

Goldoni, A. 741, 775

Goldstein, Y. 1773

Gómez-Cortéz, A. 1779

Gordillo, G. 1617, 1671, 1675, 1681, 1687, 1693

Gotter, R. 709, 937

Grad, G. 1243

Greber, T. 1243

Gregoratti, L. 413, 705, 751

Greig, D. 693

Grice, S. C. 277

Grigorashchenko, O. N. 353

Grimmer, H. 481

Grioni, M. 1117

Groh, U. 223

Groso, A. 567

Grum-Grzhimailo, A. 141

Gudat, W. 165, 1375

Guerrero-Penalva, R. 1697

Gullikson, E. M. 267, 593, 921

Gumenchuk, G. B. 353

Günster, J. 1511

Günster, S. 599, 751

Guo, G. Y. 1251

Guo, J.-H. 1369

Gurevich, Yu. G. 1703

Gutierrez, R. M. 1623

Guzmán, A. M. 1453

Gweon, G.-H. 1309

Gwo, S. 213

Hague, C. F. $811,921,977$

Hallmeier, K. H. 381

Hamilton, B. 577

Han, Z. 371

Hanf, M.-C. 1431

Hannon, J. B. 1565

Hara, T. 635

Harada, Y. 503, 515, 983

Harasawa, A. 365, 681, 769, 901, 1275,1287

Hartel, P. 223

Haruyama, Y. 769

Hase, I. 1127

Hasegawa, N. 641

Hasegawa, S. 335, 407

Hasui, S. 487

Hatano, T. 587, 943

Hatherly, P. A. 117, 129, 577

Hattori, K. 401

Hayano, H. 631

Hayashi, K. 865 
Hayoz, J. 235

Hecht, J.-D. 381

Heinäsmäki, S. 137

Heinz, K. 1413

Hergenhahn, U. 13

Hernández, M. P. 1709

Hernández-Calderón, I. 1667, 1725

Herrera-Pérez, J. L. 1645

Heun, S. 171, 413, 431

Hibma, T. 1007

Hinks, D. 1091

Hino, S. 1263

Hirai, C. 1357

Hirai, M. 313

Hiraoka, K. 1079

Hirata, G. A. 1661

Hiraya, A. 85, 503

Hirayay, A. 63

Hitchcock, A. P. 193

Hoesch, M. 1243

Hoffmann, S. V. 723

Holland, D. M. P. 577

Hong, C. K. 261, 497

Hong, I.-H. 213

Hong, K. P. 255

Hong, L. 1395

Horiba, K. 1085

Horisberger, M. 481

Horn, S. 1369

Hotta, Y. 571

Hoyer, E. 549

Hsu, L.-S. 1251

Hu, G. 371

Hu, J. 437

Huang, C. D. 1443

Huang, D. J. 1007

Huang, M. 69

Huang, S. H. 1109

Hudej, R. 775

Hüfner, S. 1275, 1281

Huhne, T. 1135

Humphrey, I. 577

Huo, D. 1035

Husain, S. 1053

Huttula, M. 117

Huttula, S.-M. 137

Hwang, C.-C. 261, 305

Hwu, Y. 567, 1109

Ibuki, T. $\quad 63,85,1351$

Ichikawa, K. 871, 1035

Ichimaru, S. 593

Ichimura, T. 1459

Igarashi, J.-I. 1197, 1203

Igeta, M. 943

Ihm, K.-W. 305

Iimori, T. $\quad 681,907$

Ikeda, N. 1327
Ikeda, T. 561

Ikeura-Sekiguchi, H. 193

Imada, S. 877, 955, 961

Imamura, M. 335

Imazono, T. 663, 669, 1097

Imbihl, R. 751

Infante, H. 1617, 1681

Ino, A. 1065

Ionel, D. 1127

Ishida, S. 955

Ishiguro, E. 401, 503

Ishii, H. 407, 425, 441, 831, 837, 1257

Ishikawa, S. 663

Ishiwata, Y. 983

Ito, E. 425,441

Ito, H. 593

Ito, K. 335

Ito, T. 1127

Itoh, M. 325

Ivanov, M. I. 347

Iwami, M. 313

Iwasaki, K. 583, 1263

Iwasaki, T. 1059

Iwazumi, T. 1059

Jacobsen, C. 185

Jagutzki, O. 93, 515

Jan, J. C. 293, 1053

Jark, W. 925

Jarvis, G. K. 129

Jastrabík, L. 473

Je, J. H. 567, 1109

Jewitt, D. E. 277

Jian, S. 1091

Jing, L. 1395

Jiyang, W. 1395

Johal, T. K. 249

Johansson, L. I. 687, 1493

Johnson, P. 1091

Johnson, R. L. 1809

Jones, S. A. 277

Journel, L. 977

Joyeux, D. 655

Juárez, H. 1715

Juárez, R. 1715

Juan, A. 1437

Juha, L. 347

Jungwirth, K. 347

Jupille, J. 949

Kachurovskaya, N. A. 1425

Kado, M. 641

Kaiser, N. 599

Kakizaki, A. 861, 865, 1275, 1287

Kaku, M. 615

Kamada, M. 325, 1297

Kamenskikh, I. A. 469
Kamezawa, C. 313

Kamimori, K. 63,85

Kanakura, N. 803

Kanashima, T. 401

Kang, T. 285

Kang, T.-H. 261, 305

Kani, Y. 341, 1315

Kanomat, T. 955

Karawajczyk, A. 117, 147

Kashiwakura, T. 1059

Katırcıŏlu, S. 1401

Kato, Y. 641

Kaulich, B. 203, 243, 413, 705

Kawachi, T. 641

Kawai, K. 561

Kawamata, S. 871

Kawamori, E. 555

Kawamura, N. 855

Kawasaki, T. 1459

Kelez, N. 549

Kempter, V. 1511

Kendziora, C. 1091

Kennedy, R. A. 129

Kevan, S. D. 1029

Khaidukov, N. M. 271, 621

Khan, N. 277

Khelifi, A. 1387

Khomenkova, L. I. 1047

Kihara, T. 365

Kijima, M. 1263

Kikas, A. 1303

Kim, B. 261, 285, 305

Kim, G. B. 261, 497

Kim, H. J. 305

Kim, K.-J. 261, 285, 305

Kim, O. H. 497

Kim, W. 931

Kim, Y. Y. 261

Kimura, A. 487, 1079, 1275, 1287, 1315, 1357

Kimura, Y. 803

Kink, M. 609

Kink, R. 609

Kinne, M. 797

Kinoshita, T. 365, 681, 769, 901, 1287

Kirm, M. 271, 299, 371, 621, 699, 783, 1291, 1333, 1363

Kirschner, J. 877

Kisand, V. 783, 1333

Kishida, H. 1065

Kishimoto, H. 1459

Kiskinova, M. 705, 751

Kitajima, M. 51

Kitakami, O. 669

Kiwata, H. 365

Kiyokura, T. 431 
Klauser, R. 213

Kleibert, A. 913

Klemm, M. 1369

Klusmann, M. 105

Knöchel, C. 177

Knop-Gericke, A. 359

Kobayashi, K. $\quad 515,529$

Koitzsch, C. 235

Kojima, K. 1079

Kolmakov, A. A. 1269

Kolobanov, V. N. 469

Komori, F. 681, 907

Kondo, Y. 521, 587

Koon, D. W. 1721

Koprinarov, I. N. 193

Korol, A. V. 1185, 1191

Korolev, V. D. 347

Korotaev, A. V. 1291

Korsunskaya, N. E. 1047

Kortus, J. 1097

Kotani, A. 849, 983, 1149

Kotani, E. 487,1287

Koyano, I. 51, 89, 93

Koyano, M. 1041

Krása, J. 347

Krasnikov, S. A. 359

Krause, M. O. 1229

Kravárik, J. 347

Krembel, C. 1431

Krisch, M. 969

Krischok, S. 1511

Krivec, R. 1161

Krug, C. 393

Krupa, J. C. 621

Kruzhalov, A. V. 1291

Kubeš, P. 347

Kubodera, S. 615

Kubota, S. 447

Kubota, Y. 555

Kubozuka, K. 93

Kuch, W. 877

Kugeler, O. 13

Kühl, S. 105

Kuhlenbeck, H. 223

Kukk, E. 117, 137

Kumar, R. 1053

Kumigashira, H. 1041, 1127

Kunze, H.-J. 627

Kuramoto, K. 267

Kurita, R. 1041

Kurmaev, E. Z. 1097, 1345

Kusakma, M. 313

Kuvaldin, E. V. 491

Kuwano, T. 803

la Rosa, S. 249, 375

La, Y.-H. 305

Laarmann, T. 111
Labis, J. 313

Lai, B. 567

Lai, W. Y. 293

Lakshmi, A. P. 39

Lalaoui, A. 1387

Lam, S. K. 271, 621

Landínez Téllez, D. A. 1791

Landazábal, F. 1671

Larabell, C. 177

Larciprete, R. 741, 775

Larramendi, E. M. 1725

Lauer, S. 57

Lavollée, M. 93

Lawrence, J. R. 193

Lazzarino, M. 413

Le Fevre, P. 949

Lebedinskaya, M. L. 491

Lee, C. $\quad 255,631,837,1257$

Lee, J. F. 293

Lee, J. M. 293

Lee, K. W. 261

Lee, M. K. 261, 497

Lee, S. F. 1109

Lee, T.-H. 213

Legros, M. 177

Leonov, N. B. 491

Leppard, G. G. 193

Lewis, B. R. 31

Li, Q. 69

Li, Z. 723

Liao, J.-D. 213

Liebel, H. 57

Lilienkamp, G. 223

Lin, J. Y. 1443

Liou, Y. 1109

Liu, H. 1655

Liu, Y. 69

Lizzit, S. 741

Lo, D. 271, 621

Locatelli, A. 171, 599, 741

Logvinov, G. N. 1703

Lopera, W. 1729

Lopez, S. 1637

López-Carreño, L. D. 1735

López-Luna, E. 1725

Lorenz, M. 353

Lozzi, L. 375

Lu, P. 641

Lu, Z. 293

Luches, P. 709, 937

Lüning, J. 437

Lürssen, B. 751

Lushchik, A. 299, 1363

Lushchik, C. H. 1363

Lushchik, Ch. 299

Lyakhovskaya, I. I. 1097

Ma, X. 69
Magnan, H. 949

Mahfoud, A. 1655

Mai, Z. H. 293

Makhov, V. N. 271, 621

Makino, H. 769

Maksimov, J. 609

Manabe, T. 1065

Manago, T. 331

Mandelzweig, V. B. 1161

Manson, S. T. 39, 1143

Many, A. 1773

Marbach, H. 751

Marburger, S. 13

Margaritondo, G. 567, 1109, 1117

Mariño, A. 1757

Mariot, J.-M. 977

Marks, S. 549

Marquette, A. 141

Marr, P. G. 277

Marsi, M. 171, 599

Martínez-Juárez, J. 1741

Martin, D. 709

Martinez, E. 1637

Martinez, J. 1715

Martinez-Lope, M. J. 1121

Martins, D. R. 1409

Martinson, I. 299, 609, 1303

Masciovecchio, C. 775

Massa, N. E. 1121

Matsuda, T. D. 831,1257

Matsui, S. 769

Matsushita, T. $843,849,961,1321$, 1327

Matthew, J. A. D. 693

Mayhew, C. A. 129

McGuinness, C. 1369

Melo, L. L. 1409

Meltchakov, E. 925

Méndez, H. 1761, 1765

Mendoza, A. 1729

Mendoza-Alvarez, J. 1645

Mertins, H.-Ch. 481, 925

Meyer, M. 141

Mikami, H. 561

Mikhailin, V. V. 469

Mikheev, L. D. 645

Mimura, K. 871, 1035

Minár, J. 1179

Minot, C. 1425

Miotti, L. 393

Mirabella, D. A. 1803

Mirone, A. 921, 977

Mitsuke, K. 583

Miyahara, T. $\quad 631,831,837,1257$

Miyamae, T. 407

Miyasaka, H. 561

Miyata, N. 663, 669, 1097 
Mizohata, H. 1035

Mizuguchi, M. 331

Mizumaki, M. 843, 849, 855, 1327

Moewes, A. 1345

Moia, T. 937

Möller, T. 111

Montejano-Carrizales, J. M. 1747

Montero, M. L. 1795, 1799

Montes, A. 1753

Moon, J. H. 305

Moon, S. 285

Morán-López, J. L. 1747

Moré, S. D. 1297

Morais, J. $\quad 393,889,895$

Morales, A. L. 1753

Moreno, A. 1761

Moreno, L. C. 1693

Morgante, A. 709, 937

Morihara, A. 487

Morin, C. 193

Morrison, G. R. 705

Moshammer, R. 93

Msezane, A. Z. 1155

Muiño, R. D. 1213

Müller-Albrecht, R. 57

Muramatsu, Y. 93, 267, 593

Muranaka, T. 1097

Muro, T. $\quad 831,961,1321$

Nagai, K. 593

Nagao, T. 1203

Nagaoka, S. 63,85

Nagasaki, F. 341, 1079, 1315, 1357

Nagashima, K. 641

Nahm, T.-U. 931

Nahon, L. 599

Naitoh, Y. 681

Nakai, S. 1059

Nakajima, H. 861

Nakamura, S. 1327

Nakanishi, T. 1297

Nakatake, M. 1357

Nakatani, T. $843,1321,1327$

Nakatsuji, K. $\quad 681,907$

Nakayama, K. 901

Nakazawa, M. 849, 855, 983, 1149, 1327

Namatame, H. 341, 487, 529, 535, 1079, 1287, 1357

Namikawa, K. 593, 631

Narimura, T. 529

Narita, H. 1287

Nasu, K. 1017

Nath, K. G. 431

Naumović, D. 235

Navarrete, L. 1757

Nee, J. B. 125

Nefedov, V. I. 1209
Negishi, H. 961

Negodin, E. $\quad 621,1333$

Nekrasov, I. A. 1345

Nelson, A. J. 387

Nery, G. 1655

Netzer, F. P. 1553

Neubauer, R. 789

Neumann, M. 1097

Newton, A. 709

Nicolay, G. 1275, 1281

Niebergall, L. 1173

Nilsson, P. O. 1275,1281

Nishikawa, Y. 1079

Nishitani, T. 1297

Niwano, M. 803

Nolting, F. 437

Nõmmiste, E. 1303

Nordgren, J. 989, 1103, 1369

Nutarelli, D. 599

Obolensky, O. I. 1191

Obu, K. 631, 831, 837, 1257

Odaka, M. 1059

Oelsner, A. 509, 883, 889, 895

Ogasawara, H. 1149

Ogawa, T. 1459

Ogino, T. 431

Ogurtsov, A. N. 45, 353

Oh, S.-J. 931

Ohashi, H. 63, 85, 401

Ohi, A. 313

Ohno, S. $\quad 681,907$

Ohtsubo, H. 837

Oji, H. 425

Okabayashi, J. 331

Okabayashi, Y. 871, 1035

Okada, K. 51, 63, 85, 89, 1023, 1065, 1351

Okada, Y. 1263

Okajima, T. 441

Okamoto, H. 401, 1065

Okamoto, J. 837

Okamoto, Y. 555

Okane, T. 1065

Oku, T. 561

Okuda, K. 871

Okuda, T. 365, 681, 769, 901

Okudaira, K. K. 335, 407

Okuyama, M. 401

Olvera, J. 1715

Olvera, J. 1741

Ono, C. K. 331

Ono, K. 171, 365, 1085

Onsgaard, J. 723

Orani, D. 249

Ortega, R. 203

Osanna, A. 185

Oshima, C. 1459
Oshima, M. 331, 1085

Ostertag, Ch. 883

Osterwalder, J. 1243

Otani, C. 561

Ottaviano, L. 675

Ouchi, Y. 425, 441

Oura, M. 515

Ozawa, K. 717

Padmore, H. 549

Paez, B. A. 1761, 1765

Paget, D. 1497

Pajasová, L. 473

Pampuch, C. 1113

Panaccione, G. 977

Paolicelli, G. 541

Paolucci, G. 741, 775

Papageorgopoulos, A. C. 1475

Papusha, V. P. 1047

Park, C. 285

Park, C.-Y. 305

Park, J. W. 305

Parmigiani, F. 541

Passacantando, M. 375

Pavlovska, A. 171

Pavlychev, A. A. 99

Peña, C. 1821

Peña, J. L. 1709

Pedio, M. 675

Peloi, M. 541

Pérez, F. 1729

Pérez-Dieste, V. 729

Perera, C. C. 593

Perera, R. C. C. 267

Pérez, F. 1729

Pérez-Dieste, V. 729

Perfetti, L. 1117

Perlov, A. 1135

Petaccia, L. 675

Pezzi, R. P. 393

Phalippou, D. 655

Piamonteze, C. 1121

Piccin, M. 249

Pinchaux, R. 1497

Plenge, J. 105

Pleslić, S. 627

Polack, F. 655

Polupan, G. P. 1047

Pong, W. F. 293, 1053

Poole, M. W. 599

Popović, D. 235

Posada, Y. 1655

Potkin, L. I. 469

Potts, A. W. 705

Präg, A. 347

Preikszas, D. 223

Preobrajenski, A. B. 359

Prieto, P. 1611, 1729, 1791 
Prince, K. C. 159

Profeta, G. 675

Proietti, M. G. 821, 1071

Pronsato, M. E. 1437

Prosperi, T. 977

Prudnikova, G. V. 1375

Pukird, S. 861

Pulci, O. 1497

Purandare, R. C. 249

Pustovarov, V. A. 1291

Qiao, S. 487, 1287

Quinn, F. M. 577

Rachlew, E. 117

Rader, O. 1113, 1375

Radtke, C. 393

Rafil, O. 1387

Ramírez-Porras, A. 1769, 1773

Ramos, A. Y. 1121

Rangel, R. 1779

Rao, K. V. R. 1053

Rata, A. D. 1007

Reginelli, A. 1117

Reichardt, G. 165

Reining, L. 1497

Renault, E. 599

Rennie, E. E. 13

Resto, O. 1655

Revcolevschi, A. 1103, 1369

Reyes, J. A. 1651, 1827

Rey-González, R. R. 1785

Richter, B. 223

Richter, R. 159

Ristau, D. 599

Rius I Riu, J. 117, 147

Roa-Rojas, J. 1791

Rockenberger, J. 437

Rodríguez, J. C. 1785

Rodríguez, P. 1453

Rodriguez, M. M. 1047

Rohlena, K. 347

Rojas, C. 1117

Rolles, D. 1213

Romberg, R. 759

Romero, M. T. 1815

Rose, H. 223

Rosei, R. 541, 741

Rotenberg, E. 1029

Rubini, S. 249

Rüdel, A. 13

Rudenkov, V. V. 353

Rudolf, M. 413

Rühl, E. 99, 105, 141

Ruocco, A. 541

Ruus, R. 1303
Ryzkov, A. V. 1339

Saalem, F. 989

Saar, A. 1303

Sabonnadiere, M. P. 645

Sacchi, M. 811, 921, 977

Sáenz, A. 1795, 1799

Saito, K. 587, 943

Saito, N. 51, 85, 93

Saitoh, T. 861

Saitoh, Y. 843, 849, 961, 1321, 1327

Sakai, O. 1035

Sakamoto, K. 1235

Sakiyama, D. 1035

Sakuma, T. 593

Sakurai, J. 1035

Salamon, K. 455

Saleh, A. 1109

Salmassi, F. 921

Salomé, M. 203

Salvadori, M. C. 1409

Sánchez, H. 1757

Sánchez, J. R. 1803

Sánchez, M. C. 821, 1071

Sánchez-Royo, J. F. 729

Sancrotti, M. 775

Sandino J. W. 1687, 1693

Santamaría, J. 1611

Santaniello, A. 937

Santucci, S. 375,675

Sasaki, J. 63, 85

Sasaki, K. 1059

Sasaki, M. 961

Sasaki, W. 615

Såthe, C. 989

Sato, H. 341, 561, 831, 1079, 1257, 1315,1357

Sato, T. 1127

Sauvage, M. 1497

Savchenko, E. V. $\quad 45,353$

Savushkin, A. V. 491

Sawada, M. 865

Schäfer, J. 1029

Schäfers, F. 481, 925

Schönhense, G. $\quad$ 509, 883, 889, 895

Schürmann, M. 735

Schürmann, M.-C. 105

Schaff, O. 223

Schaffers, K. I. 387

Scheer, M. 925

Scherbina, L. V. 1047

Schicketanz, M. 889, 895

Schieffer, P. 1431

Schlögl, R. 223, 359

Schlueter, R. 549

Schmidt, Th. 223

Schmidt-Böcking, H. 93

Schmitt, T. 1103, 1369
Schmoranzer, H. 57

Schneider, G. 177

Schwarzkopf, O. 165

Seccombe, D. P. 153

Seddon, E. A. 693

Sedo, K. 555

Seki, K. $\quad$ 407, 425, 441

Sekiguchi, T. 77

Sekiyama, A. 961, 1321

Selg, M. 609

Senba, S. 341, 1315

Sentis, M. L. 645

Senz, V. 913

Serova, A. E. 491

Sette, F. 969

Seubert, A. 1413

Seyama, A. 803

Shamin, S. N. 1345

Shamoto, S. 1127

Shanrong, Z. 1395

Shen, T.-H. 693

Sheng, Z. 69

Shi, C. S. 699

Shi, C. 371

Shi, J. Y. 699

Shi, J. 371

Shiino, O. 1085

Shikin, A. M. 1113, 1375

Shimada, K. 487, 529, 535, 1079, 1287

Shimizu, H. M. 561

Shimizu, Y. 51, 63, 85

Shimoyama, I. 77

Shin, H. J. 261, 305, 497

Shin, S. 503, 515, 983, 1275

Shinoda, M. 831, 837, 1257

Shinohara, M. 803

Shiozawa, H. 631, 837, 1257

Shirai, M. 331

Shirakawa, H. 1263

Shpinkov, I. N. 469

Shulakov, A. S. 461

Shyu, S.-G. 1007

Sirotti, F. 977

Smith, K. E. 1369

Smith-Gicklhorn, A. M. 353

Solovjev, I. A. 1185, 1191

Solov'yov, A. V. 1191

Sombrowski, E. 1333

Sorba, L. 413

Soukup, L. 473

Spangenberg, M. 693

Spassky, D. A. 469

Spector, H. 1827

Spehr, R. 223

Spezzani, C. 921

Srivastava, J. P. 1053 
Stankevitch, V. G. 1269, 1339

Stankiewicz, M. 117, 147

Starnberg, H. I. 1275

Stedile, F. C. 393

Steeg, B. 1333

Stefani, G. 541

Steier, C. 549

Stein, A. 185

Steinrück, H.-P. $\quad 789,797$

Strocov, V. N. 1275, 1281

Subías, G. 821, 1071

Suetin, N. V. 271

Suga, S. 877, 955, 961, 1221, 1321

Sugawara, H. 831, 1257

Sukegawa, K. 641

Sumitomo, M. 441

Suraban, W. 861

Susini, J. 203, 243

Suzuki, I. H. 63,85

Suzuki, S. 431

Suzuki, T. 515

Svetchnikov, N. 249

Szargan, R. 359

Taguchi, M. 1167

Taguchi, Y. 871, 1035

Tai, R. 631

Takahashi, K. 319, 1297

Takahashi, M. 587, 1197

Takahashi, N. 693

Takahashi, T. 995, 1041, 1127

Takata, Y. 503

Takatsuka, H. 521

Takayama, H. 487

Takayama, Y. $\quad 631,831,837,1257$

Takayama-Muromachi, E. 1345

Takeda, Y. 529, 1079

Takenaka, H. 593, 641

Takeuchi, N. 1641, 1809, 1815

Takeuchi, T. 983

Takizawa, Y. 561

Taleb-Ibrahimi, A. 599

Tamenori, Y. $63,85,89$

Tamine, M. 1387, 1465

Tan, K. L. 1443

Tanaka, H. 51

Tanaka, K. 319

Tanaka, M. 641

Tanaka, S. 1297

Tanaka, S.-I. 769

Taniguchi, M. 341, 487, 529, 535, 1079, 1287, 1315, 1357

Tanner, D. B. 1109

Tappe, W. 99

Tayagaki, T. 319

Tcheremiskine, V. I. 645

Terekhov, V. A. 1047

Terminello, L. 387
Themlin, J.-M. 1281

Thomas, A. G. 277

Thorne, R. E. 1029

Tjeng, L. H. 1007

Tkachenko, A. A. 651

Tokushima, T. 503

Tolentino, H. C. N. 1121

Tomita, N. 1017

Tommasini, R. 541

Torchynska, T. V. 1047

Torelli, P. 921

Torres, J. 1631, 1821

Tromp, R. M. 1565

Trovò, M. 599

Tsai, M.-H. 293

Tsai, W.-L. 567

Tsang, K.-L. 213

Tu, S. J. 125

Tuckett, R. P. $\quad$ 129, 153

Tumanov, V. I. 347

Turchini, S. 977

Tyliszczak, T. 193

Ueda, K. 21, 51, 89, 93

Ueda, Y. $\quad 341,1315,1357$

Ueno, N. 335, 407

Uhrberg, R. I. G. 1235

Ullschmied, J. 347

Umbach, E. 223

Underwood, J. H. 921

Unsworth, P. 709

Uozumi, T. 871

Urakawa, J. 631

Valeri, S. 937

Valla, T. 1091

van Buuren, T. 387

Van Hove, M. A. 1213

Vasil'chenko, E. 299

Vasil'ev, A. N. 469

Vaz, A. R. 1409

Vázquez, G. J. 1827

Verdini, A. 937

Verkhovtseva, E. T. 651

Veseth, L. 147

Vicentin, F. C. 1121

Vielhauer, S. 45, 783, 1333, 1363

Vinogradov, A. S. 359

Virojanadara, C. $\quad 687,1493$

Vivas, P. 1611

Vogt, S. 177

Vollweiler, F. 57

von Haeften, K. 111

Voronin, N. A. 491

Wabnitz, H. 111

Wada, K. 769

Walker, R. P. 599
Wang, C. R. 293

Wang, S.-C. 213

Waring, K. 31

Warren, S. 277

Watabe, C. 555

Watanabe, H. 561, 803

Watanabe, M. 503, 515, 521, 571, 587, 663, 934, 983, 1097, 1351

Watanabe, T. 1059

Watanabe, Y. 431, 555

Weber, T. 93

Wehlitz, R. 1229

Wei, D.-H. 213

Wei, Y. 371

Weightman, P. 709

Weimar, R. 759

Weiss, D. 177

Weisz, S. Z. 1773

Weisz, Z. 1655

Wells, B. O. 1091

West, J. B. 577

Westphal, C. 735

Whelan, C. M. 789, 797

Whitfield, S. B. 1229

Wilhein, T. 243

Winiarczyk, P. 117, 147

Wirick, S. 185

Woods, G. T. 1345

Wu, C.-C. 1007

Wu, W. P. 1007

Xiong, Z. T. 1443

Ya. Amusia, M. 1155, 1161, 1209

Yaji, K. 1287

Yamada, M. 681, 907

Yamaguchi, N. 555, 635

Yamamoto, M. 571

Yamamoto, S. 631

Yamane, H. 335

Yamasaki, A. 955, 961

Yamashita, M. 1065

Yamaura, T. 615

Yamazaki, T. 1059

Yanagihara, M. 663, 669, 1097, 1345

Yang, T. 293

Yao, Y. D. 1109

Yarmoshenko, Yu. M. 1345

Yarzhemsky, V. G. 1209

Yatsu, K. 555

Yin, G.-C. 213

Yokoo, A. 365

Yokoyama, T. 425

Yoshida, H. 63, 85

Yoshida, K. 313

Yoshigoe, A. 843, 1327

Yoshii, K. 855 
Yoshikawa, M. 555

Yoshimura, D. 407, 425

Young, A. T. 549

Yu, S. 69

Yu. Bakshaev, L. 347

Yu. Ivanov, V. 1291

Yu. Kirikova, N. 621

Yu. Svetchnikov, N. 375, 1269

Yu. Titov, O. 1703

Yu. Turischev, S. 1047

Yuasa, S. 1257

Yusof, Z. 1091
Zacharias, H. 735

Zadneprovsky, B. I. 469

Zaharko, O. 481

Zarate-Corona, O. 1645

Zdyb, R. 1485

Zhang, G. B. 699

Zhang, G. 371

Zhang, H. M. 1235

Zhang, T. 693

Zhang, X. Y. 699

Zharnikov, M. 213

Zhidomirov, G. M. 1425
Zhigadlo, N. D. 1345

Zhou, H. J. 699

Zhou, O. 431

Zhou, W. 129, 153

Zhou, X. 69

Zhou, Y. X. 699

Zhu, W. 431

Zhu, X.-M. 989

Ziethen, Ch. 509

Zimina, A. V. 461

Zimmerer, G. 45, 271, 371, 469, 621, 699, 783, 1291, 1333, 1363 
Abukawa, T. 499

Ahlbehrendt, D. 353

Akasaki, I. 537

Akimoto, K. 431, 519

Albano, E. V. 867

Alcock, S. G. 525

Allison, W. 249

AlShamaileh, E. 641

Amano, H. 537

An, B. 763

Aoki, T. 345

Arakawa, H. 305

Arnault, J. C. 127

Asensio, M. C. 981

Atrei, A. 861

Aubel, D. 55

Avila, J. 981

Azizian, S. 585, 895

Baher, S. 13

Bai, B. 331

Bardi, U. 861

Barlam, D. 263

Barnard, A. S. 233

Barnes, C. J. 641

Bartoš, I. 195

Benslim, N. 677

Bernhard, T. 399

Blum, R.-P. 353

Boerma, D. O. 405

Boggild, P. 963

Bolmont, D. 55

Boo, J.-H. 629, 635

Borsa, D. M. 405

Böttcher, A. 39

Bouchaud, E. 797

Bouznik, V. M. 101

Braga, R. R. 213

Braun, A. 889

Brizuela, G. 661

Brown, I. G. 1

Burghaus, U. 39, 7

Burkholder, L. 909

Caillard, J. 81

Calatayud, M. 175

Cattani, M. 1, 571, 903

Chen, G. C. 629

Cho, S.-H. 635

Chu, W.-G. 505

Chuang, T. J. 617
Clark, R. G. 415

Conrad, H. 39

Cortigiani, B. 861

Cottam, M. G. 13

Crompton, K. E. 373

Cui, K. 763

Curson, N. J. 415

Dąbrowski, J. 849

Dávila, M. E. 981

de Izarra, C. 81

Derry, T. E. 295

Diani, M. 55

Diouri, J. 55

Dmytruk, A. 283,289

Dorfman, S. 213, 227

Du Plessis, J. 365

Emoto, T. 431

Fansuri, H. 549

Finlayson, T. R. 373

Fisher, P. 277

Fissel, A. 849

French, D. 549

Fu, X. 325, 381

Fuks, D. 213, 227

Fukui, Y. 467

Furukawa, Y. 543

Gallego, J. M. 405

Gao, J. 317

Gard, F. S. 669

Gartstein, E. 263

Geng, Z. 95

Glander, G. S. 105

Gobal, F. 895

Gonzalez, E. A. 879

Gonzalez, E. 661

Goto, S. 543

Gotoh, Y. 271, 425

Grachev, S. Y. 405

Grey, F. 963

Gu, N. 569

$\mathrm{Gu}$, S. L. 625

Hahn, P. H. 163

Hallam, T. 415

Hanatani, K. 271

Hannech, E. B. 677

Hansen, T. M. 963
Hasegawa, S. 963

Hata, A. 431

Hatanaka, Y. 345

Held, G. 487

Hester, J. 513

Higano, M. 337

Hirao, T. 611

Hisanaga, T. 305

Hoadley, J. 241

Hobara, R. 963

Hofmann, K. R. 605

Holst, B. 249

Honda, S.-I. 611

Hong, I.-H. 617

Hongo, S. 467

Honma, K. 499

Horii, S. 431

Howes, P. B. 525

Hsu, Y.-J. 617

Hu, A. 311

Huan, A. C. H. 493

Huang, F. 95

Huang, L. 569

Huang, X. Q. 311

Hughes, A. E. 365

Hung, A. 169

Hwang, C. S. 591

Ichimiya, A. 431

Ikuno, T. 611

Iloukhani, H. 585

Irurzun, I. M. 23

Ishii, H. 257, 505

Ishikawa, T. 543

Ito, A. 721

Ito, M. 543

Itoh, A. 697

Jakovidis, G. $\quad 443,533$

Jamieson, D. 365

Jamieson, I. M. 443

Jamieson, I. 533

Jasen, P. V. 879

Jeong, J. 121

Jiang, Q. 49

Jiang, S. S. $\quad 311,317$

Jin, T. 283

Johansson, L. 499

Juan, A. 661, 879

Jung, C.-K. 635 
Kaburagi, M. 189

Kaito, C. 361, 435, 455, 461

Kamada, K. 611

Kanagawa, T. 963

Kang, B. S. 183

Kang, H. J. 183

Kang, M. 189

Kasapoglu, E. 737

Kasuya, A. 305

Katayama, M. 611

Katırcığlu, Ş. 577

Kawaguchi, T. 449

Kawanowa, H. 271, 425

Kawata, H. 431, 519

Keeffe, M. 925

Kido, O. 361, 461

Kido, Y. 389

Kim, H. J. 591

Kim, I. G. 207

Kim, T.-K. 635

Kimura, Y. 361, 455

King, D. A. 23

Kirchner, C. 373

Kitada, M. 473

Klauser, R. 617

Klemradt, U. 373

Kondrikov, N. B. 101

Kono, S. 499

Krupski, A. $\quad 65,843$

Kubler, L. 55

Kulkarni, G. U. 87

Kuryavy, V. G. 101

Kurzina, I. 861

Lahtinen, J. 641

Lamoudi, N. 677

Lang, M. 117

Lapeyre, G. J. 925

Lau, D. C. 277

Lay, G. L. 981

Leckey, R. 669

Lee, B. C. 207

Lee, J. I. 207

Lee, M.-N. 635

Lee, S.-B. $\quad 629,635$

Lerotholi, T. J. 487

Li, J.-Q. 691

Liao, J. 569

Lim, S. $\quad 121,685$

Lin, H. 283

Liu, K. 381

Liu, Y. M. 311

Long, J. M. 365

Lu, D. Q. 625

Ma, S. 831

Ma, U. 95

MacLaren, D. A. 249
Maehara, Y. 425

Mak, D. K. 649

Makau, N. W. 295

Makhloufi, B. 677

Matsuda, I. 963

Matsukawa, H. 449

Matsushita, T. 543

McCulloch, D. G. 365

Melo, L. L. 1, 571, 903

Menzel, D. 487

Mguig, B. 175

Minot, C. 175

Miranda, R. 405

Mochizuki, T. 543

Mogilyanski, D. 263

Mola, E. E. 23

Monetti, R. 867

Monteiro, O. R. 1

Morgan, M. 513

Morgenstern, M. 933

Mortimer, E. C. 241

Moshfegh, A. Z. 745

Mróz, S. 65, 843

Mudie, S. 513

Müller, B. H. 605

Munakata, M. 499

Mundim, K. C. 213

Mundim, K. 227

Nagashima, S. 337

Nagel, O. A. 879

Naitoh, M. 473

Nakamura, M. 543

Niehus, H. 353

Nihei, Y. 257, 505

Ning, H. 95

Nishigaki, S. 473

Nishitani, R. 305

No, S. Y. 591

Nozoye, H. 917

Oberbeck, L. 415

Oh, S. K. 183

Ohashi, H. 543

Ohata, T. 543

Ohkura, S. 611

Okazawa, T. 389

Oshima, C. 697, 721

Otsuka, I. 337

Oura, K. 611

Ovechko, V. 289

Owari, M. 257, 505

Papageorgopoulos, C. A. 73

Paterson, P. J. K. 365

Pavlov, K. 513, 533

Peng, R. W. 311

Petersen, C. L. 963
Petrakov, A. 533

Pfandzelter, R. 117, 399

Pham, G. H. 549

Pistonesi, C. 661

Plessis, J. D. 241

Puisto, S. R. 487

Punegov, V. 533

Qiu, F. 311

Radny, M. W. 201

Rao, G. R. 917

Rawle, J. L. 525

Read, M. N. 481

Riley, D. J. 249

Riley, J. D. 669

Rokuta, E. 697, 721

Rotenberg, E. 925

Rout, B. 365

Rovida, G. 861

Russo, S. P. 233, 241

Sainio, J. 641

Saito, A. 543

Saito, Y. 361, 435, 455, 461

Sakamoto, Y. 467

Sakata, O. 543

Saltas, V. 73

Salvadori, M. C. 1, 571, 903

Sari, H. 737

Sasaki, H. 305

Sato, T. 361,435

Schattke, W. 195

Schmidt, W. G. 163, 221

Schofield, S. R. 415

Seino, K. 221

Seitz, M. 373

Sha, H. 317

Shchitovskaya, E. V. 101

Shen, B. 625

Shen, H. 569

Shevlyuga, V. 861

Shi, H. 201

Shi, Y. 625

Shimizu, R. 505

Shimomura, M. 499

Shimura, T. 543

Shin, J. C. 591

Shiraki, I. 963

Shiraki, S. 257, 505

Shoji, F. 473

Simmons, M. Y. 415

Simon, L. 55

Singh, A. 443

Sirghi, L. 345

Smith, P. V. 201

Snook, I. K. 169, 233

Souda, R. 271 
Spencer, M. J. S. 169

Stacchiola, D. 909

Stonham, A. 365

Strasser, T. 195

Sugiyama, H. 431, 519

Sumitani, K. 519, 543

Sumiyama, K. 305

Sun, C. Q. 1009

Sun, Y. Y. 493

Suzuki, H. 361, 455

Sökmen, I. 737

Tabuchi, M. 513, 537

Tajima, A. 721

Tajiri, H. 431, 519, 543

Takahashi, S. 543, 611

Takahashi, T. $431,519,543$

Takahasi, M. 543

Takeda, Y. 513, 537

Takeshita, K. 543

Takeuchi, J. 467

Tamine, M. 727

Tamura, K. 257, 505

Tan, W. S. 317

Tanabe, F. 963

Tanaka, T. 697,721

Tanigaki, T. 361,455

Tanikawa, T. 963

Tateishi, Y. 305

Teichert, C. 837

Toh, S. K. 365

Tong, S. Y. $\quad 831,925$

Toyama, N. 473

Tysoe, W. T. 909
Ueba, H. 771

Ueno, H. 361

Urano, T. 467

Urazgil'din, I. 117

Uruga, T. 543

Usher, B. F. 669

Vallée, O. 81

Vasilyeva, M. S. 101

Vaz, A. R. 571

Vicente, J. L. 23

Vickers, R. E. M. 277

Vijayalakshmi, S. 87

Vinod, C. P. 87

Wang, C. R. 605

Wang, F. 625

Wang, X. 325, 381

Wang, Y. 95

Wee, A. T. S. 493

Wei, D.-H. 617

Wibawanta, S. 549

Widstrand, S. M. 499

Winter, H. 117, 399

Wu, H. S. $\quad 831,925$

Wu, X. S. 317

Xie, H. 751

Xiu, X. Q. 625

$\mathrm{Xu}, \mathrm{L} . \quad 569$

Xu, M. 837

Xu, S. H. 831, 925

Xu, Y.-J. 691
Yamashita, K. 697

Yang, J.-R. 331

Yang, L. 317

Yaoita, M. 337

Yarovsky, I. 169

Yazawa, T. 283

Yin, G.-C. 617

Yong, K. 121

Yong, K. 685

Yoshimura, M. 467

Yu, Y. 381

Yu, Z. X. 831

Yuan, C. 763

Zakeri, K. H. 745

Zhang, X. 519

Zhang, D. K. 549

Zhang, P.-C. 331

Zhang, R. 625

Zhang, T.-J. 763

Zhang, X. 431

Zhang, Y.-F. 691

Zhang, Y. 569

Zhao, D. S. 49

Zhao, M. 49

Zhao, Z. 325, 381

Zheng, Y. D. 625

Zhou, H. W. 751

Zhou, S.-Q. 331

Zhu, J. 95

Zou, J.-S. 331 
Abdallah, M. S. 427

Abdel-Rahman, M. A. 427

Abouelhassan, S. 83, 199

Adhikari, S. $553,559,585$

Adhikary, S. 553, 559, 585

Ahonen, M. 457

Arabski, J. 563

Atuchin, V. V. 191

Azizi, A. 563

Badawi, E. A. 427

Badawi, E. 167, 451

Bartoš, I. 447

Belhadi, M. 87, 99, 321

Belykh, S. F. 391

Bhavsar, S. R. 443

Bilić, A. 185

Borca, C. N. 531

Boumrar, H. 155

Bozzolo, G. 235, 591

Brongersma, H. H. 341

Burghaus, U. 521

Canto, G. 77

Canzian, A. 235

Carabineiro, S. A. C. 385

Cattani, M. 223, 283, 463

Ch. Argirusis 419

Chadli, R. 321

Chai, J. C. 217

Chen, G. 15

Chen, Q. 305

Chen, X. 15

Cheng, J. 27

Cheng, X. 211

Chou, W.-C. 229

Creemers, C. 341

Dai, C. Y. 433

Dai, X. Q. 1

Dang, H. 111

Dashti, A. 137

Dashti, M. 33

de Groot, F. M. F. 385

Deng, D. 599

Desai, C. F. 443

Dew, S. K. 391

Dinia, A. 563

Dong, S. 205

Dowben, P. A. 531

Du, W. 379, 491
Duan, C.-G. 531

El-Mansy, M. K. 199

Elmansy, M. 83

Fan, D. B. 27

Farías, D. 591

Farias, R. J. C. 223

Foulias, S. D. 419

Ganesan, V. 265

Gasmi, A. 337

Geng, Y. L. $71,379,491$

Gesari, S. B. 145

Gunes, M. 49

Güneş, M. 403

Guo, L. X. 367

Guo, L. 291, 497

Gupta, V. K. 41

Han, J. 211, 503

Hannech, E. B. 337

Hardt, D. E. 217

Hasegawa, N. 553

He, Z. 7

Helfensteyn, S. 341

Hirsimäki, M. 457

Huang, S. Y. 515

Hush, N. S. 185

Iqbal, K. 117

Irurzun, I. M. 57

Jeong, H.-K. 531

Jiang, B. L. 367

Jiang, D. 205

Jiang, S. 433

Jimbo, T. $553,559,569,577,585$

Joshi, K. P. 41

Juan, A. 145

Junell, P. 457

Kamaratos, M. 419

Kasapoglu, E. 49, 403, 411

Kato, S. 553

Kesler, V. G. 191

Kh. Zakeri 137

Khan, K. M. 117

Khater, A. 87, 99

Khirman, M. A. 259

King, D. A. 57

Kjeldgaard, L. 385

Komesu, T. 531
Kumar, U. 451

Lacharme, J.-P. 21

Lam, Y. C. 217

Lamoudi, N. 337

Lei, T. Q. 367

Lei, T. 291, 497

Li, D. Y. 173

Li, H. 211

Li, J.-S. 509

Li, Q. 111,503

Li, T. B. 71

Li, W. 173

Li, Y.-R. 509

Lim, G. C. 305

Lin, J. S. 229

Liu, H. Y. 379, 491

Liu, J. 531

Liu, R. 291, 497

Lu, M.-W. 331

Luyten, J. 341

Mao, L. 111

Maschke, K. 87

Mei, W. N. 531

Meng, H. 599

Miao, Y. 7

Miranda, R. 591

Mishra, R. K. 271, 541, 607

Misra, K. D. 271, 541, 607

Mokutani, H. 553

Mola, E. E. 57

Mosca, H. O. 235

Moshfegh, A. Z. 33

Mukhopadhyay, P. K. 451

Murukeshan, V. M. 217, 259

Ng, V. M. 515

Nieuwenhuys, B. E. 385

Omer, A. M. M. $553,559,585$

Ozturk, E. 297

Pagura, C. 391

Palmstrøm, C. J. 531

Pan, D. 503

Pan, Y. 433

Pandey, R. P. 607

Park, B. 179, 373

Peng, C. 485, 599

Petukhov, A. G. 531

Pokrovsky, L. D. 191 
Pronsato, M. E. 145

Qin, X. 205

Rafil, O. 155

Rafti, M. 57

Rathore, M. K. 41

Rathore, V. 41

Read, M. N. 447

Reimers, J. R. 185

Romero, M. T. 77

Rubensson, J.-E. 385

Rusop, M. 553, 559, 569, 577, 585

Ryu, Y. 373

Salman, F. 83, 199

Salvadori, M. C. 223, 283, 463

Sari, H. 49, 297, 403, 411

Schultz, B. D. 531

Schurmans, M. 341

Sheha, E. 83, 199

Shrivastava, S. B. 41

Sinha, O. P. 265

Soga, T. $\quad 553,559,569,577,585$

Sökmen, I. 49, 297, 403, 411

Song, S. 485, 599

Soni, P. H. 443

Srivastava, P. C. 265

Stepanova, M. 391

Sun, D. L. $\quad 71,379,491$
Takeuchi, N. $\quad 77$

Tamine, M. 155

Tan, J. L. 259, 305

Tiwari, R. P. 541, 607

Tolstogouzov, A. 391

Tong, S. Y. 1

Tran, D. V. 217

Uchida, H. $\quad 553,559,585$

Umeno, M. $\quad 553,559,569,577,585$

Valden, M. 457

Vaz, A. R. 223

Vázquez de Parga, A. L. 591

Vicente, J. L. 57

Vlachos, D. 419

Voronkova, V. I. 191

Wang, B. 15

Wang, G. 15

Wang, H. 27

Wang, J. 503, 521

Wang, X. C. 305

Wang, X. Q. 71, 379, 491

Wang, Y. L. 491

Wang, Y. M. 367

Wang, Z. 211, 503

Wu, H. S. 1

Xie, M. H. 1
$\mathrm{Xu}$, D. $\quad 71,379,491$

$\mathrm{Xu}, \mathrm{M} . \quad 515$

$\mathrm{Xu}, \mathrm{S} . \mathrm{H} .1$

$\mathrm{Xu}, \mathrm{S}$. Y. 515

Yan, H. 27

Yang, C. 211, 503, 509

Yang, H. 211

Yang, J. 363

Yanovskii, V. K. 191

Yesilgül, U. 411

Yin, W.-G. 531

Yong, K. 179, 373

Yoshimura, M. 27

Yu, S. 7

Yu, W.-F. 509

Yu. Maklakova, N. 191

Zaïbi, M.-A. 21

Zhang, G. H. 71, 379, 491

Zhang, S.-M. 311

Zhang, T. 363

Zhang, X. 7

Zhang, Y. C. 27

Zhang, Z. 111

Zhao, J. 15

Zheng, H. Y. 217, 259, 305

Zhou, Y. C. 433 


\section{CUMULATIVE AUTHOR INDEX (Volume 12)}

Abdallah, M. S. $\quad 197,203,463$

Abdel-Latif, R. M. 197

Abdel-Rahman, M. A. 197, 203, 463

Abdulrazaq, O. A. 299

Adachi, M. 19

Adhikari, S. 13

Adhikary, S. 13

Avilés, F. 101

Azizi, A. 391

Badawi, E. A. $\quad$ 1, 197, 203, 463

Boumrar, H. 85

Cattani, M. 221

Ceh, O. $\quad 101,289$

Chai, J. C. 351

Chen, C. 401

Chen, C. Z. $\quad 41,123,161,209,369$, 443

Chen, G. 279, 417

Corona, J. E. 289

Dai, S. 59

Dai, Z. 397

Ding, P. 75

Dinia, A. 391

Drofenik, M. 97, 239

Du, Z. 59

Fang, Z. 355

Fenineche, N. 391

Filardo Bassalo, J. M. 221

Fort, T. 457

Gao, L. 355

Gao, S. 433

Geng, Y. L. 413

Gesari, S. B. 227

Ghodsi, F. E. 425

Gu, S. 409

Han, J. 409

Huang, S. 397

Huo, Q. H. 41, 161, 209, 443

Irigoyen, B. L. 227

Ismail, R. A. 299
Ji, Q. 369

Jiang, X. 59

Jimbo, T. $13,19,27,35,167,173$, $185,325,333,343$

Juan, A. 227

Kang, H. 355

Kasapoglu, E. 155

Kawano, H. 107

Kinugawa, T. 27

Lam, Y. C. 351

Lan, J. 75

Lei, T. Q. $\quad 41,123,161,209$, 443

Li, Y. 59

Liu, S. $\quad 7,319$

Long, J. 397

Lu, M.-W. 67

Lu, Y. 355

Ma, G. 409

Maldonado, R. D. 289

Mishra, R. K. 47

Misra, A. P. 47

Misra, K. D. 47

Mominuzzaman, S. M. 35

Monteiro, P. J. M. 233

Oliva, A. I. 101,289

Omer, A. M. M. 13

Rafil, O. 85

Riveros, H. G. 289

Rusop, M. 13, 19, 27, 35, 167, 173, $185,325,333,343$

Ryou, J. 233

Sahari, A. 391

Salvadori, M. C. 221

Sari, H. 155

Schmerber, G. 391

Shi, J.-J. 433

Soga, T. 13, 19, 27, 35, 167, 173, $185,325,333,343$

Sökmen, I. 155

Song, S. 457

Sun, Y. 397
Tak, Y. 215

Tamine, M. 85

Tan, S. 75

Tian, Y. S. $\quad 41,123,161,209,369$, 443

Tigrine, R. 85

Tripathi, A. K. 47

Uchida, H. 13

Umeno, M. 13, 35

Uskoković, V. 97, 239

Wang, D. 59, 401

Wang, D. Y. 41, 123, 161, 209, 369,443

Wang, X. Q. 413

Wang, Z. 409

Xu, D. 413

Xu, S. 397

Yahya, K. Z. 299

Yakovkin, I. N. 449

Yang, C. 409

Yang, D. 355, 409

Yang, H. 355

Yang, J. 355

Yang, Y. 409

Yin, M. 355

Yong, K. 215

Yu, G. W. 413

Zhang, G. H. 413

Zheng, H. Y. 351

Zheng, J. 355

Zhang, L. 433

Zhang, S. $\quad 115,145,359,379$

Zhang, T. 355

Zhang, W. 7

Zhang, X. 59

Zhang, X. P. $\quad 279,417$

Zhang, Y. 457

Zhao, Y. 401

Zhou, Z. 75

Zhu, H. 351 